\title{
Management Approach for Microscopic Polyangiitis: A Case Report
}

\author{
Mohammed Al Azzawi ${ }^{1 *}$, Alfarooq Alshaikhli ${ }^{2}$, Mustafa Altaei ${ }^{1}$, Abbas Alshami ${ }^{1}$, Alsadiq Alhillan ${ }^{3}$, Asseel \\ Albayati $^{1}$ and Eric Costanzo ${ }^{4}$ \\ ${ }^{1}$ Department of Internal Medicine, Jersey Shore University Medical Center, USA \\ ${ }^{2}$ Department of Internal Medicine, University of Texas/Rio Grande Valley, USA \\ ${ }^{3}$ Hepatology/Liver transplant, Baylor College of Medicine, USA \\ ${ }^{4}$ Department of Pulmonary and Critical Care, Jersey Shore University Medical Center, USA
}

\begin{abstract}
Microscopic polyangiitis is a small vessel necrotizing vasculitis. It usually exhibits its manifestation through inflammation on the kidneys, it also presents with an extrarenal systemic involvement such as the lungs, musculoskeletal systems, central or peripheral nervous system, and skin manifestations. Laboratory markers show an elevation in the inflammatory markers and Myeloperoxidase-Antinuclear Cytoplasmic Antibody. Kidney involvement usually manifests in the form of rapidly progressive pauciimmune glomerulonephritis. The Lung present at the range of fleeting local infiltrates to massive hemorrhage is the setting of diffuse alveolar hemorrhage. Patients generally respond appropriately to induction therapy with high IV pulse-dose prednisolone plus an additional immunosuppressive agent followed with maintenance therapy with an oral immunosuppressive drug, the response to the guidelines-recommended regimen is significant in most of the patients and results in symptoms recovery concomitantly. Notwithstanding, we present a case of a female with a recent diagnosis with microscopic polyangiitis confirmed with a kidney biopsy, achieved complete remission with the combination of prednisone and cyclophosphamide. While she was on induction therapy, she developed a major relapse in the form of diffuse alveolar hemorrhage and needed an aggressive form of management in the form of plasmapheresis, prednisone, and rituximab to stabilize her condition.
\end{abstract}

KEYWORDS: Cyclophosphamide; Microscopic polyangiitis; Plasmapheresis; Rituximab; Vasculitis

ABBREVATIONS: MPA: Microscopic Polyangiitis; IV: Intravenous; CYC: Cyclophosphamide; RTX: Rituximab; SVC: Systemic Vasculitis; DAH: Diffuse Alveolar Hemorrhage

\section{INTRODUCTION}

Small vessel vasculitis is divided into three diseases: Granulomatosis with Polyangiitis (Wegener's), Eosinophilic granulomatosis with polyangiitis (Churg-Strauss), and microscopic polyangiitis (MPA). Treatment of MPA differs based on the disease severity and usually started with a remission therapy with a combination of an Intravenous (IV)prednisolone and another immunosuppressive agent i.e., cyclophosphamide (CYC), or Rituximab (RTX) preferably for 3-6 months followed by maintenance therapy for 12-24 months with a single oral immunosuppressive agent preferably RTX, other alternates are available. However, some patients may require an aggressive form of management with

Quick Response Code: $\quad$ Address for correspondence: Mohammed Al Azzawi, Department of Medicine, Jersey Shore University Medical Center, USA

Received: May 29, 2021 Published: June 04, 2021

How to cite this article: Mohammed Al A, Alfarooq A, Mustafa A, Abbas A, Alsadiq A, et. al. Management Approach for Microscopic Polyangiitis: A Case Report. 2021- 3(3) OAJBS.ID.000290. DOI: $10.38125 / O A J B S .000290$ 
plasmapheresis, biological agents, or a combination of multiple agents $[1,2]$. Establishing suitable management is challenging and varies accordingly. In this illustration, we will discuss the management guidelines and recommendations in patients of MPA, their prognosis, and the follow-up strategies.

\section{BACKGROUND}

Systemic vasculitis (SVC) comprises for diseases that are unpredictable in outcomes. The diagnosis of SVC requires a combination of clinical findings, radiological data, and histological studies. Management of the disease is challenging in the means of assessing the disease's severity, drug selections, monitoring of clinical findings, and risk for relapse. The prompt Selection of medications regimen varies among patients and require cautious evaluation of patients' risk factors and concurrent morbidities [1].

\section{METHOD}

A literature review was performed out to identify the clinical trials that is done to exert therapeutic guidelines to manage MPA patients. Our review was through conducting a search of the PubMed database and ClinicalTrials.gov using the search keywords "Microscopic polyangiitis"," ANCA-Vasculitis" "Small-vessel vasculitis". We also matched them with keywords "remission", "Induction", "Maintenance", "Relapse"," remission" and "Therapy". The results were filtered by choosing "clinical trial" option on the databases. We included only original manuscripts whom the participants have an established diagnosis of MPA enrolled in the trial. We exclude the literature reviews, systematic review, and observational studies. We also excluded clinical trials that no MPA diagnosed patients were participated. We evaluated each clinical trial in terms of Enrolled criteria, regimen used, primary and secondary outcomes, major relapses, adverse events among lines of management. Finally, we obtained written, informed consent from our patient for the publication of the details of our case.

\section{CASE PRESENTATION}

A 77-year-old female with a past medical history of Hypertension, gastroesophageal reflux disease, hyperlipidemia, ischemic heart disease, celiac disease, and diabetes mellitus. She admitted to the hospital by her primary care physician with a clinical finding of generalized fatigue, 12-pound unintentional weight loss, hematuria, and back pain for a duration of 3 weeks. Her medications included Metoprolol, atorvastatin, metformin, and aspirin. There was no family history of vasculitis or connective tissue disease. She also denies smoking, excessive alcohol intake, and illicit drug abuse. Laboratory workup revealed proteinuria at the presentation of 2.4 gram/24 hour (normal $<0.1$ gram/24-hour, elevated erythrocyte sedimentation rate, creatinine level was $3.03 \mathrm{mg} / \mathrm{dl}$ (normal range, 0.84 to 1.21 ), circulating serum MPO-ANCA level was 41.3 $\mathrm{IU} / \mathrm{ml}$ (normal range, $\leq 3.5 \mathrm{IU} / \mathrm{ml}$ ). The patient denies a recent intake of propylthiouracil, hydralazine, allopurinol, penicillamine, minocycline, rifampicin nor experimenting with any illicit drugs. Afterward, a renal biopsy performed and revealed crescent shape glomerulonephritis with associated tubular atrophy, interstitial fibrosis, and inflammation which confirmed the diagnosis of MPA. Eventually, the patient started a remission therapy with IV pulse dose methylprednisolone at a dose of $10 \mathrm{mg} / \mathrm{kg}$ per day for three days with tapered dose to $60 \mathrm{mg}$ oral prednisolone per day and oral CYC at a dose of $1.5 \mathrm{mg} / \mathrm{kg}$ per day. In a period of one week of hospital admission, her renal function shows an improvement in creatinine level of $2.38 \mathrm{mg} / \mathrm{dl}$, her symptoms resolved. Additionally, we started her on trimethoprim-sulfamethoxazole for Pneumocystis Jiroveci pneumonia prophylaxis. She was discharged for a regular follow-up every two weeks continuing prednisolone and CYC.

Four weeks later, she presented to the emergency room due to symptoms of shortness of breath, chest tightness, subjective fever, and hemoptysis. She denies any urogenital or other systemic involvement. Vital signs were remarkable for pulse oxygenation of $82 \%$ on room air, respiratory rate of 21 breaths/minute, the temperature of 97.3-degree Fahrenheit, a heart rate of 78 beats/ minutes. Her height was $167 \mathrm{~cm}$, and the weight was $61.4 \mathrm{~kg}$.

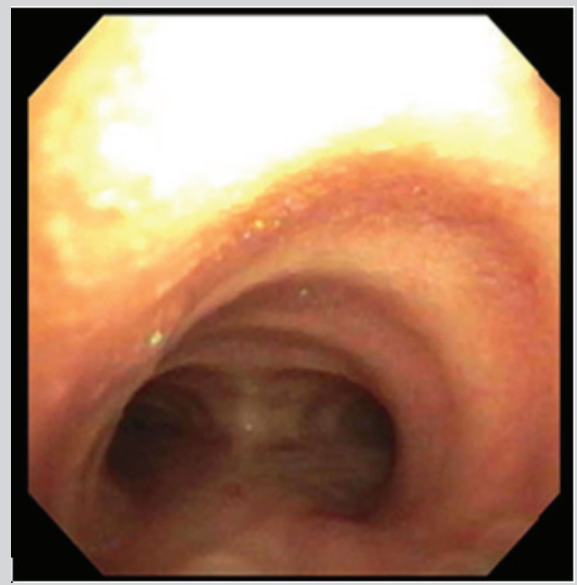

Figure 1: Tracheal rings and bifurcation showing and no signs of hemorrhage.

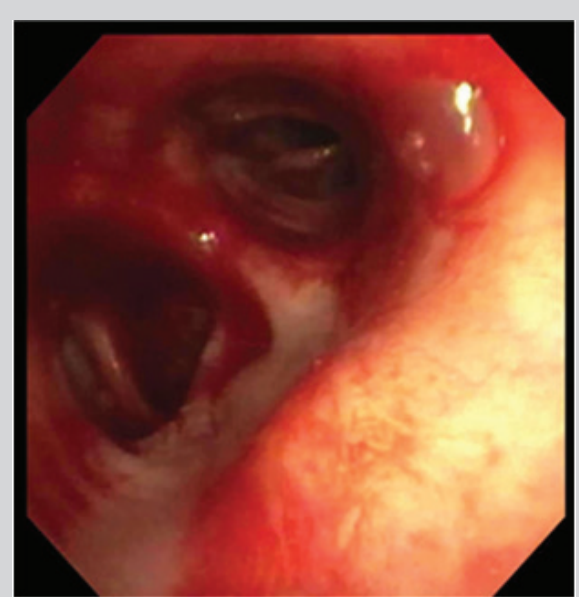

Figure 2: Left main bronchus showing extensive hemorrhage.

Upon physical exam, chest auscultation revealed bilateral inspiratory rales at mid and lower lung fields that were not present on the most recent discharge examination. The other physical examination was not notable. Chest radiograph showed diffuse airspace infiltrate in the perihilar distribution bilaterally (Figure 1). CT scan of the chest revealed a diffuse, patchy bilateral ground-glass opacity (Figure 2). laboratory finding was remarkable for creatinine level of $2.51 \mathrm{mg} / \mathrm{dl}$. Work-up was negative for systemic lupus erythematosus, antiphospholipid syndrome, and scleroderma, Tests for antinuclear antibodies and anti-glomerular basement membrane antibodies were negative. Bronchial wash culture, acidfast bacilli came back negative for an ongoing infectious process. Purified protein derivative skin test and sputum smears and cultures for acid-fast bacilli were negative. Blood and urine cultures were 
sterile and urine Histoplasma antigen, serum brucella, tularemia, Leptospira, coccidioidomycosis, and blastomycosis antibody tests were negative. Tests for human immunodeficiency virus, hepatitis $\mathrm{A}, \mathrm{B}$ and $\mathrm{C}$ viruses were also negative.

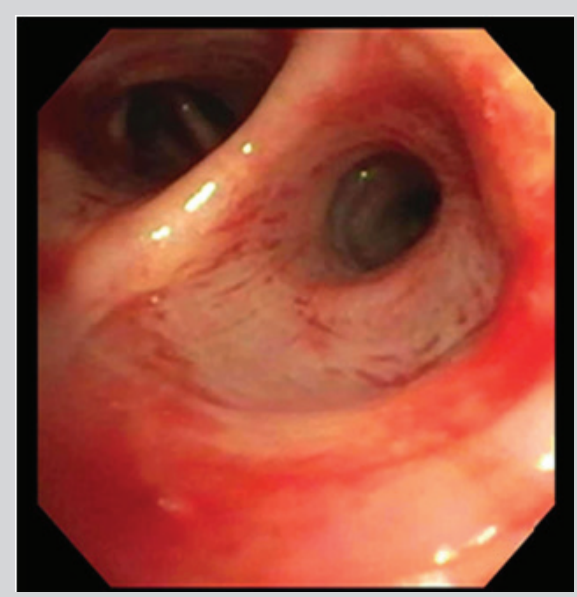

Figure 3: Left upper lobe revealing erythema and signs of hemorrhage.

The patient was transferred to the Intensive care unit (ICU)and underwent bronchoscopy that showed blood in the airways diffusely within the lung lobes consistent with diffuse alveolar hemorrhage (DAH) (Figure 3). Eventually, the patient considered to have a major relapse and received seven sessions of plasmapheresis, together with a combination of IV pulse steroids at a dose of $10 \mathrm{mg} / \mathrm{kg}$ per day for three days and RTX daily at a dose of $375 \mathrm{mg} / \mathrm{m}^{2}$ for 1 month, CYC was discontinued. Her symptoms improved and lung examination became clear. Treatment was well tolerated with good effect. Thereafter, the creatinine level after two weeks of management continues to be stabilized at $2.38 \mathrm{mg} / \mathrm{dl}$. She was discharged with a close-up follow-up plan with induction therapy with oral prednisolone and RTX for the next 6 months with regular check for complete blood count, urine routine microscopic examination, and serum creatinine. Drugs were gradually decreased to only $10 \mathrm{mg}$ orally of prednisolone without relapse within 4 months from DAH attack.

\section{DISCUSSION}

MPA is a rare small-sized non-granulomatous necrotizing vessel, may involve medium-sized vessels and has a predilection for capillaries, venules, and arterioles. It had a predilection for white, older adults. However, it could occur in non-whites children and young adults. Usually presents in the form of rapidly progressive glomerulonephritis, DAH appears in $12 \%-29 \%$ of patients [1-3]. Patients usually present with general symptoms such as fatigue, fever, muscle aches, and weight loss. Kidney involvement such as proteinuria, hematuria, followed by renal function deterioration and rapidly progressive glomerulonephritis. Lungs causes cough which could dry or productive, dyspnea, hemoptysis and wheezing. Skin findings such as palpable purpura, livedo reticularis, urticaria or digital ischemia. Neurological manifestation in the form of peripheral neuropathy, seizures and even stroke. Recurrent sinusitis, uveitis, pericarditis, and other systemic findings might also be the presenting symptoms. MPA patients are MPO-ANCA positive in $90 \%$, other are negative or rarely positive proteinase 3ANCA $[2,3]$.
The French Vasculitis Group initiated the Five Factor Score (FFS) which asses the prognosis is patients with vasculitis. Five factors to be considered in assessing patients.

a) Age above 65 .

b) Renal insufficiency (Creatinine $>1.7 \mathrm{mg} / \mathrm{dL}$ ).

c) Gastrointestinal involvement (bleeding, perforation, infarction, or perforation)

d) Cardiomyopathy.

e) Absence of ENT manifestation (presence associated with a better outcome).

Each accorded one point with a result (0,1, and 2). The mortality rate for scores 0,1 , and $\geq 2$ is $9 \%, 21 \%$, and $40 \%$ respectively. The FFS role in medications decisions has not yet been established [4]. Treatment of these patients started with the assessment of severity which influences the choice of therapy within patient-specific factors [5]. The patients are categorized according to their clinical findings into: Patients with organ or life-threatening patterns of disease i.e., rapidly progressive glomerulonephritis, pulmonary hemorrhage, cerebral vasculitis in the form of stroke, progressive neuropathy, scleritis, GI bleeding, and cardiac disease (pericarditis, myocarditis) $[5,6]$.

Stages of therapy are broken into induction and maintenance therapy:

Induction therapy: The goal is to rapidly suppress inflammation to prevent permanent organ damage. It continues for about 3-6 months. Glucocorticoids are the cornerstone of management. For patients with an organ of a life-threatening condition, studies suggested a combination of two medications with pulse IV prednisolone and either CYC or RTX. The combination of prednisolone and CYC was first described by Anthony Fauci and Sheldon Wolfe, complete remission can be achieved in $90 \%$ of patients. Although this regimen is effective, it carries a significant adverse effect associated [7,8]. No clinical trial show that either CYC or RTX is superior to each other and which to be considered as initial therapy. However, patients' profile and risk factors concern such as previous CYC usage, fertility issues, and malignancy; RTX is preferred. If either CYC or RTX been used as the primary induction therapy but did not achieve complete remission, consider either switch to the other or use a combination of both in addition to steroids. For patients who were treated with both CYC and RTX and still suffering from relapses, it is recommended to give mycophenolate mofetil as adjuvant therapy [8-10].

The use of plasmapheresis is usually reserved for patients with MPA with DAH, in rapidly progressive glomerulonephritis (Creatinine above $4.0 \mathrm{mg} / \mathrm{dl}$, and those who require dialysis), and in patients with concomitantly positive anti-glomerular basement membrane. Immunoglobulin may be prescribed at a dose of $2 \mathrm{~g} / \mathrm{kg}$ for 2 to 5 days, particularly in patients with active infection [11]. Maintenance therapy: It usually lasts for about 12-24 months with an oral immunosuppressive agent preferably RTX. Azathioprine, methotrexate, and mycophenolate mofetil are possible alternatives and may be preferred based on other patient-specific factors intended to extend remission and prevent relapse. The maintenance therapy usually started based on the initial drug used; It usually started 2-4 weeks following the last doses of CYC or after 4-6 months if RTX was used as induction therapy [12] 
Several clinical trials are done to show the differences among these medications; The (MAINTRANS) clinical trial showed that RTX is superior to azathioprine, with a 5-year rate of sustained remission and a better survival rate. The (IMPROVE) trial showed that azathioprine shows less relapses than Mycophenolate Mofetil as maintenance therapy, but both showed similar side effects. Another trial was the (WEGENT) trial which Compared azathioprine versus methotrexate, which showed both were nonsuperior to each other and similar in adverse events. In pregnancy, azathioprine is preferred as it has the lowest risk profile. Patients typically assessed after maintenance therapy ended. If symptoms vanished; discontinue the therapy. Otherwise, if symptoms or signs persist, therapy may be continued indefinitely [13-15].

Patients with non-severe disease in the absence of organ or life-threatening condition, include those with rhinosinusitis, arthritis, and/or pulmonary nodules. These patients do not require aggressive form of treatment to achieve remission. They usually show a good response to the combination of Methotrexate, and corticosteroids. However, if Glomerular filtration rate below $60 \mathrm{ml} /$ $\min / 1.73 \mathrm{~m}^{2}$. Alternatives such as azathioprine or RTX are available. The regimen usually taken for a period of a longer than 12 months $[1,6]$.

The patient's response should be assessed accordingly, and each evaluated on an individual basis. Patients achieved Complete remission which means an absence of active clinical findings. Nevertheless, the blood values do not need to return to baseline.
Even if there is persistent proteinuria or even slowly deteriorating renal function. While partial remission is considered difficult to be defined as it refers to the stabilization of laboratory markers and disappearance of extrarenal manifestation with the presence of dysmorphic red blood cells in the urine with or without red blood cells casts. It is a smoldering process that could lead to progressive organ deterioration followed by irreversible damage and usually indicated the necessity for additional therapy. In the lung, it is extremely difficult to detect whether it achieved complete or partial remission as a regular CT scan is usually done [16-18].

Relapse: Defined as the restoration of symptoms and considered the main problem in MPA patients as it increases morbidity and mortality in terms of permanent organ damage and increase drug cumulative toxicity levels. More than $50 \%$ of patients experience a relapse affecting similar or different organs from the original. If a major relapse occurs while the patient is on maintenance therapy, instate an induction therapy should be considered [8]. While, a minor relapse occurs (sinusitis), titrate the glucocorticoids up to the previous dose when complete remission achieved for 4 weeks followed by subsequent weaning, or start another immunosuppressant if it occurs more frequently. It is now theorized that female, African American, T-cell activation level, MPO-ANCA status, genetic background, and presence of ongoing infections show increase in the risks to relapse and resistant to medical therapy $[6,18]$ Management guidelines is summarizing in (Figure 4).

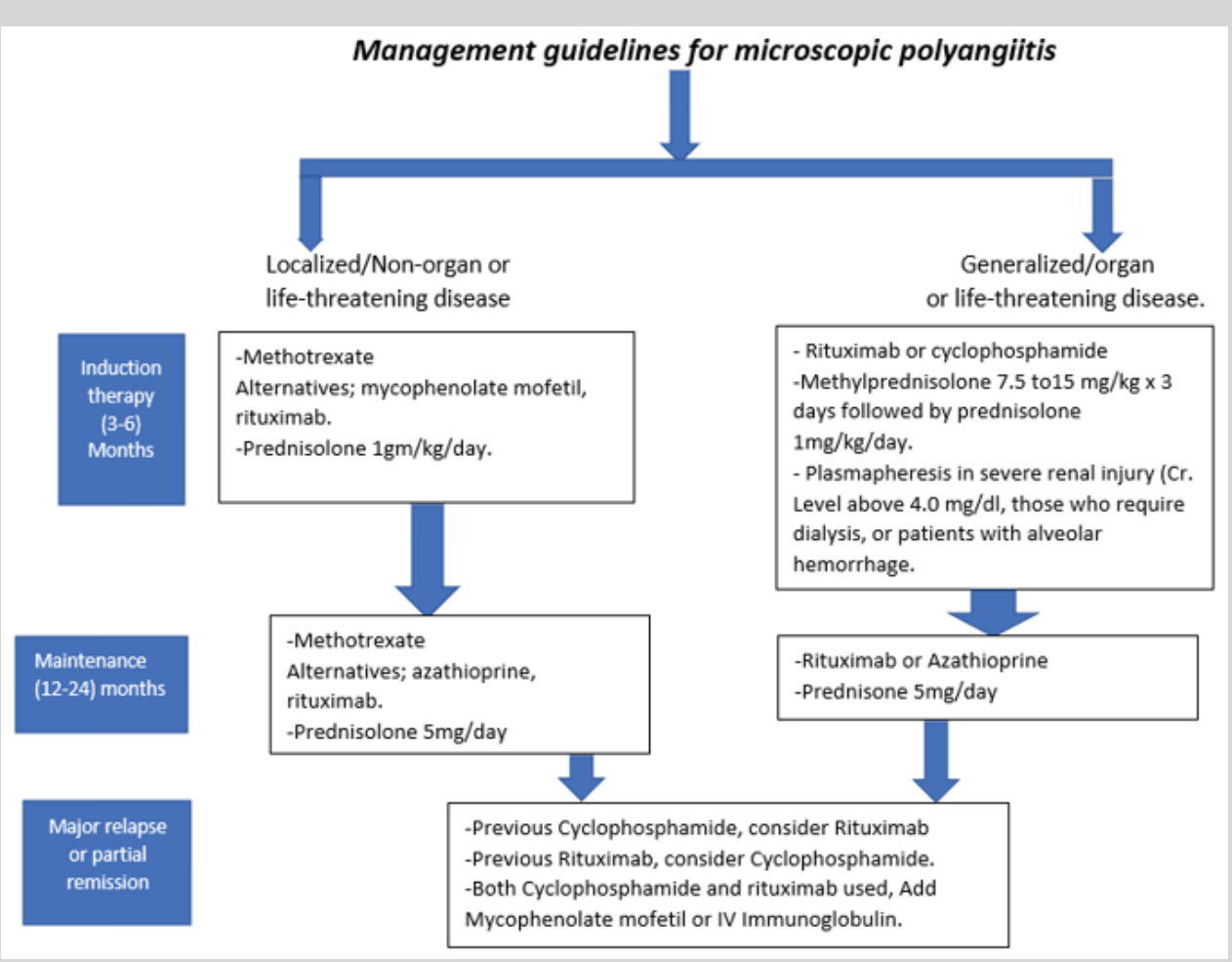

Figure 4: Management guidelines for microscopic polyangiitis.

\section{Medications and Side Effects}

Glucocorticoids: Halts the function and numbers of $\mathrm{T}$ and B lymphocytes causing immunosuppression. Used in mostly all patients with an active form of the disease. In the case of organthreatening or life-threatening disease; methylprednisolone is used and given in pulses at a dose of 7.5 to $15 \mathrm{mg} / \mathrm{kg} /$ day for the first 3 days. Following these days or as maintenance therapy, prednisolone is given at a dose of $1 \mathrm{mg} / \mathrm{kg} /$ day for one month with a gradual weaning scheme up to $5-10 \mathrm{mg} /$ day at 6 months. While in the case with non-organ involvement or non-life-threatening form of MPA, we typically started at a dose of $0.5 \mathrm{mg} / \mathrm{kg} /$ day to be tapered 
gradually. Major side effects (SEs): Hypertension, diabetes mellitus, arrhythmias, GI bleeding, cataract, glaucoma, avascular necrosis of bone, and osteoporosis [2,19].

Cyclophosphamide: is an Alkylating agent that exerts its effect via covalent binding and crosslinking of DNA, RNA, and proteins which impairs DNA replication and transcription ultimately lead either to cell death or to altered cellular function. Both IV and oral regimens are comparably effective. CYCLOPS protocol is usually used as a dose of $15 \mathrm{mg} / \mathrm{kg} /$ pulse IV three times within the first month in two weeks intervals followed by every three weeks for the next 3-6 months until complete remission is achieved. It also theorized that IV therapy had the advantages of low toxicity level, lower rates of leukopenia and infection but a higher rate of relapse. If an IV pulses regimen is used, physicians maintain adequate hydration with 2-4L given and MESNA with it to prevent hemorrhagic cystitis. While orally, it is usually given at a dose of 1.5 to $2 \mathrm{mg} / \mathrm{kg}$ daily. Both IV and oral regimens need to be titrated down in elderly patients or patients with impaired kidney function. The white blood cell counts should be monitored regularly to avoid leukopenia. Major SEs: Bone marrow suppression, Infections, Gonadal toxicity, Hemorrhagic cystitis, Bladder carcinoma, Syndrome of inappropriate antidiuretic hormone, and Myelodysplasia [20-21].

Rituximab: is a monoclonal antibody that works through depleting CD20-positive B cells. The dose of $375 \mathrm{mg} / \mathrm{m}^{2}$ in a week for a duration of 1 month as induction therapy as defined by RITUXVAS and RAVE trials or the regimen used for rheumatoid arthritis of $1 \mathrm{~g}$ then another $1 \mathrm{~g}$ in two weeks. As for maintenance therapy, used 500mg infusion every 6 months up to 2 years (4 infusions) as defined by the MAINRITSAN trial. Viral serologies (HIV, HCV, and HBV) should be tested prior to its use. RTX can be used in HIV patients if CD4>50. Concomitant usage of entecavir/ tenofovir usually recommended in patients with HBsAg and/ or anti-HBc positive patients usually continued for at least 18 months of the last RTX dose. Major SEs: Progressive multifocal leukoencephalopathy, Infusion reactions, Opportunistic infections, and Late-onset neutropenia $[9,13,22]$.

Methotrexate: inhibits dihydrofolate reductase that aids in the conversion of dihydrofolate to tetrahydrofolate, required for DNA synthesis. Usually initiated at the dose of $15 \mathrm{mg}$ /week titrated up to a target dose of $20-25 \mathrm{mg} /$ week within 3 months used specifically in the non-severe form of MPA. Folic acid at a dose of $1-2 \mathrm{mg} /$ day or Folinic acid at a dose of $5-10 \mathrm{mg} /$ day usually given to reduce toxicity. Major SEs: Hepatotoxicity, Stomatitis, Pneumonitis, and Bone marrow suppression [20].

Azathioprine: works as an inhibitor of purine metabolism, results in inhibition of DNA, RNA, and protein synthesis. The dose started at a dose of $50 \mathrm{mg} /$ day and titrated up to reach a target of $2.5 \mathrm{mg} / \mathrm{kg} /$ day. Major SEs: GI symptoms, bone marrow suppression, and recurrent infection, malignancy, and infertility. Toxicity increased with the concomitant use of xanthine oxidase inhibitors [23].

Mycophenolate mofetil: works as an inhibitor of inosine monophosphate dehydrogenase led to halt T-cell and B-cell proliferation and prevent the production of cytotoxic T-cells and antibodies. The dose given is $2-3 \mathrm{~g} /$ day. In the MYCYC trial, Mycophenolate was as effective as CYC in patients with a non-lifethreatening disease with no serious organ damage, particularly in MPO positive patients, Nevertheless, it carries a higher rate of relapse. Major SEs: GI symptoms, Bone marrow suppression, Infections, and malignancy [24].

Prognosis: There is a significant improvement in the management of MPA over the past 40 years and the survival rate reached $80 \%$ within 10 years after disease onset. Frequent relapses and chronic organ damage persist to occur. Factors associated with a higher rate of relapse are age $\geq 65$ years and DAH at diagnosis [11]. Infections due to immunosuppressant drugs considered to be the major cause of death in addition to chronic renal failure, and pulmonary failure. Recurrent DAH may be complicated with pulmonary fibrosis and lead to permanent lung damage. Renal injury may be to cause chronic kidney disease and lead to dialysis and/or transplantation. Estimates that MPA has a higher incidence of malignancy in the form of skin cancer, hematological malignancy, and solid malignancies [25].

Follow-up visits: The patients need a close monitoring plan, even during periods of remission, visits usually scheduled every two to four weeks following hospital discharge for the first three months and then every two to three months in the following months. These visits are mainly to assess the patient's response, medications side effects, performing a detailed history and physical examination, monitoring creatinine level, urinary sediment, and inflammatory markers $[19,20]$. Other tests may be required in accordance with patients' symptoms such as CT chest in patients with tracheal stenosis or/and pulmonary involvement. Also, audiograms should be performed in patients with any form of hearing difficulty. Supplement therapy with Vitamin D 600-800IU/day and daily calcium $1000-1200 \mathrm{mg} /$ day intake should be taken regularly as patients carry high risk of osteoporosis with continuous steroids intake. In addition, Dual Energy X-ray Absorptiometry (DEXA)scan is performed regularly $[25,26]$.

Opportunistic infections and vaccination: The risk for opportunistic infections reached about $50 \%$ especially within the first year of therapy, thus, it is strongly recommended that the patients received age-appropriate vaccinations such as influenza, pneumococcal and herpes Zoster. It is also recommended to administer prophylaxis against pneumocystis jiroveci while patients on induction therapy, mostly used drugs are Trimethoprim/ sulfamethoxazole at a dose of $800 / 160 \mathrm{mg}$ three times/week. Dapsone, atovaquone and pentamidine are alternatives $[25,26]$.

\section{CONCLUSION}

The pathogenesis of MPA is rapidly evolving as many strategies is considered such as molecular genotyping, usage of monoclonal antibodies and proteomic to reach a more effective and less toxic management strategies through the course of the disease. Physicians should be vigilant as MPA has high manifold presentations and has a risk of decline in clinical outcomes even after achieving complete remission. The involvement of a Multidisciplinary team such as a pulmonologist, nephrologist, gastroenterologist, hematologist, otolaryngologist, and neurologist are needed in the management of severe organs specific manifestations of MPA. Finally, we recommend reporting similar cases with a variable response to guideline-recommended therapy which enriches the anecdotes as trials are limited and mostly does not take extrarenal involvement or patient-specific risk factors into considerations.

\section{CONSENT}

The patient described in the case report had given informed consent for the case report to be published. 


\section{REFERENCES}

1. Kitching AR, Anders HJ, Basu N, Brouwer E, Gordon J, et al. (2020) ANCAassociated vasculitis. Nat Rev Dis Primers 6(1): 71.

2. Qasim A, Patel J (2021) ANCA positive vasculitis. In: Stat Pearls, Treasure Island.

3. Sacoto G, Boukhlal S, Specks U, Flores SLF, Cornec D, et al. (2020) Lung involvement in ANCA-associated vasculitis. Presse Med 49(3): 104039.

4. Guillevin L, Pagnoux C, Seror R, Mahr A, Mouthon L, et al. (2011) The Fivefactor Score revisited: assessment of prognoses of systemic necrotizing vasculitides based on the French vasculitis study group (FVSG) cohort Medicine (Baltimore) 90(1): 19-27.

5. Hellmich B (2019) ANCA-assoziierte vaskulitiden: State of the art. Z Rheumatol 78(6): 518-528.

6. Misra DP, Naidu GS, Agarwal V, Sharma A (2019) Vasculitis research: Current trends and future perspectives. Int J Rheum Dis 22(Suppl 1): 10-20.

7. Pagnoux C, Quéméneur T, Ninet J, Diot E, Kyndt X, et al. (2015) Treatment of systemic necrotizing vasculitides in patients aged sixty-five years or older: results of a multicenter, open-label, randomized controlled trial of corticosteroid and cyclophosphamide-based induction therapy. Arthritis Rheumatol 67(4): 1117-1127.

8. Ozaki S (2007) ANCA-associated vasculitis: diagnostic and therapeutic strategy. Allergol Int 56(2): 87-96.

9. Jones RB, Furuta S, Tervaert JW, Hauser T, Luqmani R, et al. (2015) Rituximab versus cyclophosphamide in ANCA-associated renal vasculitis: 2-year results of a randomized trial. Ann Rheum Dis 74(6): $1178-1182$.

10. Miloslavsky EM, Specks U, Merkel PA, Seo P, Spiera R, et al. (2013) Clinical outcomes of remission induction therapy for severe antineutrophil cytoplasmic antibody-associated vasculitis. Arthritis Rheum 65(9): 2441-2449.

11. Walsh M, Merkel PA, Peh CA, Szpirt W, Guillevin L, et al. (2013) Plasma exchange and glucocorticoid dosing in the treatment of anti-neutrophil cytoplasm antibody associated vasculitis (PEXIVAS): Protocol for a randomized controlled trial. Trials 14: 73.

12. Terrier B, Darbon R, Durel CA, Hachulla E, Karras A, et al. (2020) French recommendations for the management of systemic necrotizing vasculitides (polyarteritis nodosa and ANCA-associated vasculitides). Orphanet J Rare Dis 15(Suppl 2): 351.

13. Jain S, Chattopadhyay A, Sharma A (2020) Long-term rituximab use to maintain remission of antineutrophil cytoplasmic antibody-associated vasculitis. Ann Intern Med 173(11): 947.

14. Hiemstra TF, Walsh M, Mahr A, Savage CO, De Groot K, et al. (2010) Mycophenolate mofetil vs azathioprine for remission maintenance in antineutrophil cytoplasmic antibody-associated vasculitis: a randomized controlled trial. JAMA 304(21): 2381-2388.
15. Puéchal X, Pagnoux C, Perrodeau É, Hamidou M, Boffa JJ, et al. (2016) Long-term outcomes among participants in the WEGENT trial of remission-maintenance therapy for granulomatosis with polyangiitis (Wegener's) or Microscopic Polyangiitis. Arthritis Rheumatol 68(3): 690-701.

16. Berti A, Dejaco C (2018) Update on the epidemiology, risk factors, and outcomes of systemic vasculitides. Best Pract Res Clin Rheumatol 32(2): 271-294.

17. Monti S, Quinn KA, Christensen R, Jayne D, Langford C, et al. (2020) Use and reporting of outcome measures in randomized trials for antineutrophil cytoplasmic antibody-associated vasculitis: a systematic literature review. Semin Arthritis Rheum 50(6): 1314-1325.

18. Berti A, Specks U (2019) Remission maintenance in ANCA-associated vasculitis: does one size fit all? Expert Rev Clin Immunol 15(12): 12731286

19. Biedroń G, Włudarczyk A, Wawrzycka AK, Wójcik K, Sznajd J, et al. (2020) Treatment and its side effects in ANCA-associated vasculitides - Study based on POLVAS registry data. Adv Med Sci 65(1): 156-162.

20. Maritati F, Alberici F, Oliva E, Urban ML, Palmisano A, et al. (2017) Methotrexate versus cyclophosphamide for remission maintenance in ANCA-associated vasculitis: A randomised trial. PLoS One 12(10): e0185880.

21. De Groot K, Rasmussen N, Bacon PA (2005) Randomized trial of cyclophosphamide versus methotrexate for induction of remission in early systemic antineutrophil cytoplasmic antibody-associated vasculitis. Arthritis Rheum 52: 2461-2469.

22. Smith RM, Jones RB, Specks U, Bond S, Nodale M, et al. (2020) Rituximab as therapy to induce remission after relapse in ANCA-associated vasculitis. Ann Rheum Dis 79(9): 1243-1249.

23. Gopaluni S, Smith RM, Lewin M (2017) Rituximab versus azathioprine as therapy for maintenance of remission for anti-neutrophil cytoplasm antibody-associated vasculitis (RITAZAREM): study protocol for a randomized controlled trial. Trials 18: 112 .

24. Tuin J, Stassen PM, Bogdan DI, Broekroelofs J, Van PP, et al. (2019) Mycophenolate mofetil versus cyclophosphamide for the induction of remission in nonlife-threatening relapses of antineutrophil cytoplasmic antibody-associated vasculitis: randomized, controlled trial. Clin J Am Soc Nephrol 14(7): 1021-1028.

25. Biedroń G, Włudarczyk A, Wawrzycka AK, Wójcik K, Sznajd J, et al. (2020) Treatment and its side effects in ANCA-associated vasculitides - Study based on POLVAS registry data. Adv Med Sci 65(1): 156-162.

26. Samson M, Puéchal X, Devilliers H, Ribi C, Cohen P, et al. (2014) Longterm follow-up of a randomized trial on 118 patients with polyarteritis nodosa or microscopic polyangiitis without poor-prognosis factors. Autoimmun Rev 13(2): 197-205. 